\section{$\underset{\substack{\text { hommes } \\ \text { \& migrations }}}{ }$}

\section{Hommes \& migrations}

Revue française de référence sur les dynamiques

migratoires

1289 | 2011

Les frontières du sport

\title{
Grandir et travailler en France
}

Jouer pour l'équipe nationale algérienne de football dès 1980

\section{Stanislas Frenkiel}

\section{Q OpenEdition \\ 1 Journals}

\section{Édition électronique}

URL : http://journals.openedition.org/hommesmigrations/801

DOI : 10.4000/hommesmigrations.801

ISSN : 2262-3353

Éditeur

Musée national de l'histoire de l'immigration

\section{Édition imprimée}

Date de publication : 1 janvier 2011

Pagination : 80-91

ISSN : 1142-852X

\section{Référence électronique}

Stanislas Frenkiel, « Grandir et travailler en France », Hommes \& migrations [En ligne], 1289 | 2011, mis en ligne le 31 décembre 2013, consulté le 14 novembre 2019. URL : http://journals.openedition.org/ hommesmigrations/801; DOI : 10.4000/hommesmigrations.801 


\section{Grandir et travailler en France}

\section{Jouer pour l'équipe nationale algérienne de football dès 1980}

Par Stanislas Frenkiel, docteur ATER, Centre de recherche et d'innovation sur le sport (EA 647)

Les déterminants sportifs, politiques, sociaux, économiques et culturels qui incitent les footballeurs professionnels descendants d'immigrés algériens en France à s'engager dans l'équipe nationale algérienne dès 1980 restent à analyser. Car le "désir d'Algérie" chez les footballeurs "algériens de France" ne répond pas seulement à des objectifs sportifs.

Ce désir de jouer provisoirement de l'autre côté de la Méditerranée peut se comprendre en partie par les discriminations réelles et ressenties dans le pays où ces joueurs ont grandi. 
Les fondements sociaux, économiques, culturels, politiques et géographiques de la construction du projet migratoire à la période coloniale et post-coloniale des "migrants sportifs", à savoir "ceux qui bougent avec la balle, ceux qui migrent avec l'intention de gagner leur vie en jouant au football ${ }^{11}$ ", sont désormais bien connus ${ }^{(2)}$. En ce sens, ils s'inscrivent dans un débat plus large relatif à l'ethnicité et surtout à l'intégration par le sport ${ }^{(3)}$. Pourtant, puisqu'ils participent également à ce débat et qu'ils sont peu connus, les déterminants qui incitent les descendants d'immigrés africains à s'engager dans les équipes nationales de leur pays d'origine doivent être identifiés. Afin d'interroger les migrations sportives vers les équipes nationales africaines - et non les modalités d'accès au haut niveau et la professionnalisation de joueurs qui connaissent tous une forme d'ascension sociale grâce au football -, nous prenons le parti de nous focaliser sur une population sportive bien particulière. Il s'agit des footballeurs professionnels algériens évoluant dans le Championnat de France de première et de seconde divisions, qu'ils soient "nés en France" ou qu'ils y aient grandi ("migrants familiaux" venus durant leur enfance en France avec leurs parents).

D'une part, parce que, en tant que fils d'immigrés ayant principalement fui la misère et l'ordre socio-racial et la guerre d'Algérie(1954-1962), ces 93 footballeurs "algériens de France" constituent de 1968 à 2002 la plus importante cohorte de joueurs ayant leurs racines en Afrique. Ils devancent de loin les Français d'origines marocaine, sénégalaise et camerounaise, ce qui correspond à l'étiage des flux migratoires globaux de ces quatre territoires vers la France. D'autre part, parce que parmi ces 93 footballeurs "algériens de France", 43 (soit 46,23 \%) choisissent l'équipe nationale algérienne (les Verts ou les Fennecs), alors que seulement 4 (soit 4,3\%) évoluent durant leur carrière sportive en équipe de France de football. Leur attirance manifeste pour l'équipe nationale du pays où sont nés leurs parents - même si, il faut le retenir, ils ne souhaitent ni s'y installer ni y vivre - doit évidemment être interrogée. Il nous faut donc étudier précisément les conditions sportives, politiques, sociales, économiques et culturelles en France et en Algérie qui suscitent chez les footballeurs "migrants familiaux" et "nés en France" ce "désir d'Algérie", un pays dans lequel ils n'ont pas grandi et où parfois même ils ne sont pas nés. Conditionnée par de multiples facteurs, leur appartenance nationale algérienne se concrétise notamment au sein de leur engagement en équipe nationale algérienne. C'est donc la construction de ce "désir d'Algérie" que nous allons analyser ici. Au-delà de l'étude de la simple expérience émotionnelle et d'une stratégie de réussite, nous mettrons en lumière l'idéalisation du miroir algérien. Prendre en compte et dépasser trois présupposés s'avère nécessaire.

Le premier présupposé consiste à penser que le fait de posséder en France la nationalité algérienne - en vertu du "droit du sang" - les pousserait spontanément à vouloir jouer avec les Fennecs. De plus, les footballeurs "algériens de France" seraient obsédés par 
l'idée de devenir un jour international algérien. Le stoppeur Nordine Kourichi, né en 1954 à Ostricourt, footballeur professionnel de 1976 à 1986 et international algérien de 1980 à 1986, reconnaît qu' 'il y avait une vie en cité qui était vachement agréable, mais entre 10 et 15 ans, je ne pensais pas à l'Algérie. Je me sentais comme quelqu'un qui vivait avec des parents qui étaient immigrés en France, c'est tout". Enfin, le troisième présupposé renvoie au fait que la frustration engendrée par le "boycott" entre 1968 et 1980 des footballeurs professionnels en équipe nationale algérienne créerait à elle seule naturellement chez eux le désir de s'y investir. En effet, il faut savoir qu'après l'indépendance de l'Algérie, aucun footballeur professionnel n'a pu évoluer en équipe nationale algérienne depuis l'échec des Verts face à la Tunisie le 29 décembre 1968. Mais dans un contexte où, à partir du début des années quatre-vingt, de nombreuses équipes nationales africaines s'appuient chaque année davantage sur leurs professionnels, l'État algérien autorise pour la première fois certains joueurs professionnels algériens à rejoindre les Fennecs le 28 décembre 1980 (match SoudanAlgérie [1-1] à Khartoum). Dans un pays de moins en moins dominé par l'idéologie socialiste et traversé par une crise économique et de nouvelles lignes de fracture comme le meurtrier "printemps berbère" de mars-avril 1980, "mettre toutes les chances de victoire de son côté et symboliser la politique de réinsertion sélective de l'émigration encouragée par l'État (4)" sont les deux principales raisons sportives et extra-sportives qui permettent d'expliquer leurs nouvelles convocations.

\section{Au-delà des motifs socio-économiques et sportifs}

Ayons tout d'abord présent à l'esprit que malgré quelques gratifications (billets d'avion, primes...), les footballeurs professionnels ne s'enrichissent pas avec les Verts. Ils perçoivent déjà mensuellement dans leurs équipes françaises des salaires conséquents le plus souvent en hausse d'une saison à l'autre, ce qui leur donne de nouvelles prérogatives et des loisirs "select". D’après Omar Kezzal, président de la Fédération algérienne de football (FAF) à trois reprises, "les indemnités, pour un joueur professionnel opérant et touchant des salaires en France, c'est de la rigolade". Pourtant, les footballeurs professionnels algériens, même si très peu le reconnaissent, ont des intérêts économiques à devenir internationaux. En dépit du fait que nous n'avons pas eu accès aux contrats des footballeurs professionnels, être international dans l'une des meilleures équipes africaines du début des années quatre-vingt pèse sans aucun doute indirectement dans les négociations des mensualités des joueurs et dans leur valeur marchande et d'échange. 
Pour comprendre leurs choix sportifs, il s'avère indispensable d'appréhender, à la fin des années soixante-dix, le renouveau de l'équipe de France de football sous l'impulsion de Michel Hidalgo. Autour de footballeurs comme Luis Fernandez, Alain Giresse, Michel Platini, Jean Tigana et Marius Trésor, l'équipe de France progresse. Avec de jeunes joueurs prometteurs issus pour certains des récents centres de formation (1973), elle enchaîne d'exceptionnelles performances internationales. En équipe de France, les places et les positions, autrement dit "les postes", sont occupées par des joueurs de très haut niveau et restent relativement stables d'une grande compétition à l'autre. Les footballeurs "migrants familiaux" et "nés en France" contournent majoritairement ce marché de l'équipe de France saturé et concurrentiel et s'engagent préférentiellement avec les Fennecs, afin de participer eux aussi à des joutes internationales - qui plus est dans une équipe reconnue comme l'une des meilleures du continent africain. C'est ce que reconnaît comme d'autres Nordine Kourichi, le premier footballeur algérien "né en France" invité à rejoindre les Verts le 28 décembre 1980 : "On m'aurait appelé en équipe nationale française, j’y aurais été. C'est la Fédération algérienne qui m'a appelé la première et je me suis retrouvé en sélection là-bas et puis c'est tout."

Alors que depuis 1964 la Fédération internationale de football association (FIFA) institue l'obligation pour un footballeur à la double nationalité de jouer pour une seule équipe nationale, les footballeurs algériens "migrants familiaux" et "nés en France" seraient donc animés d'une affection profonde envers l'équipe nationale algérienne : motivations socio-économiques et sportives, certes, mais aussi symbole d'un véritable attachement au pays d'origine de leurs parents. Outre le fait que ces "enfants du béton" - selon l'expression de Zaïr Kédadouche, né à Tourcoing en 1957, professionnel de 1975 à 1986 - obtiennent tous la nationalité algérienne à leur naissance en vertu du "droit du sang", leur "identité algérienne" et son façonnement en France doivent désormais être appréhendés d'un point de vue social et culturel.

\section{Le façonnement familial et socioculturel de l'fridentité algérienne"}

Appuyons-nous sur les recherches de Nacira Guenif Souilamas ${ }^{(5)}$ et intéressons-nous au rôle déterminant des familles de ces footballeurs qui vivent dès l'enfance une "socialisation primaire" vis-à-vis de l'Algérie. Cette éducation véhicule normes et valeurs et leur rappelle constamment les liens culturels puissants qui les unissent au pays d'où sont originaires leurs parents.

Transmise et apprise au sein de la cellule familiale (la majorité des mères des footballeurs s'occupent du foyer familial et ne parlent pas français), la langue arabe confronte les 
futurs footballeurs à leurs racines algériennes. Presque tous la maîtrisent et elle façonne leur identité.Écoutons l'analyse de Gilbert Grandguillaume pour qui “ dans ce rapport de l'individu à la langue, il y a ainsi à la fois imposition d'une loi et investissement d'un désir(')". L'attaquant Chérif Oudjani, licencié depuis son enfance au Racing Club de Lens, ville dans laquelle il voit le jour en 1964, s'exprime à ce sujet : "traditions, fêtes religieuses, repas familiaux : jai tout de suite su que mes parents n'étaient pas là depuis vingt générations". Linculcation de la foi et de la morale musulmane (leur pratique est vécue comme modérée) dans un pays traditionnellement chrétien et laïc vient renforcer ce

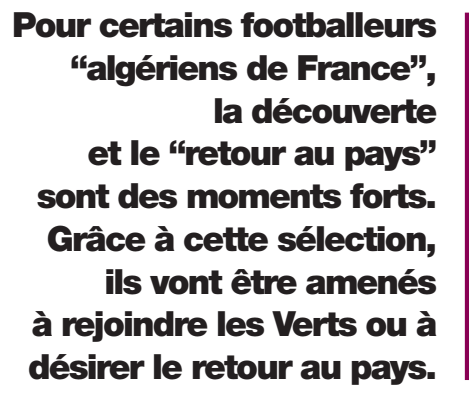
sentiment d'être un Algérien en France. Pour Fathi Chebel, né en 1956 à Lyon et international algérien de 1981 à 1986, "on était élevé sur les principes musulmans. Mes parents n'étaient pas spécialement pratiquants à l'époque mais bon, il y a le respect des traditions". Au-delà de cette transmission familiale de la langue arabe ou berbère et de la religion musulmane, l'islam façonne de nouveaux hexis corporels ${ }^{(7)}$ et devient une "référence symbolique et un instrument d'identification sociale ${ }^{(8)}$." Les récits parentaux souvent "nostalgiques" sur l'Algérie, "le bled" abandonné, sont nombreux et s'accompagnent d'ailleurs d'un discours classique et illusoire sur le retour providentiel dans le pays des origines. Un "mythe du retour" qui, d'après Abdelmalek Sayad, "entretient le sentiment du provisoir ${ }^{(9)}$.'. Leurs parents fréquentent de façon quasi exclusive des amis musulmans, et plus précisément des Algériens. Ces comportements s'inscrivent dans la tradition des centaines de milliers d'Algériens qui connaissent eux aussi "le déracinement" et l'exil. En France, ce façonnement familial et socioculturel de l'identité algérienne passe par une éducation - stricte et traditionnelle - qui s'appuie sur la langue arabe ou berbère, l'islam, l'évocation du "pays", la fréquentation d'amis musulmans et le sentiment de faire partie d'une minorité. Ce lien avec l'Algérie se raffermit également lors des séjours estivaux. En effet, en Algérie, le plus souvent, à l'occasion des vacances d'été, tous les futurs internationaux algériens découvrent dès l'enfance le pays d'origine de leurs parents.

C'est donc non seulement en France mais aussi en Algérie que s'édifie socialement et culturellement leur identité algérienne. Mettre en évidence ce façonnement familial - associé à une excellence sportive construite dans les clubs professionnels français - permet donc d'appréhender leurs motivations et ambitions à vouloir jouer pour l'Algérie, ou tout au moins à répondre positivement aux sollicitations de 
l'équipe nationale algérienne. Et ce d'autant plus qu'ils ont conscience du poids familial qui repose sur eux : ils pressentent des attentes (parfois affichées) sur leurs engagements sportifs susceptibles de provoquer la fierté ou au contraire la déception, voire un soupçon de traîtrise... D'après Nordine Kourichi, comme tant d'autres, "mes parents étaient tellement fiers que je joue en équipe nationale algérienne et que je fasse le Mundial. C'était tout à fait honorifique pour eux". Fathi Chebel ajoute que devenir international français, "ça aurait jeté un froid dans la famille". Cependant, leurs parents et familles ne sont pas les seuls responsables de leur conviction d'être des "Algériens de France", des "Français pas comme les autres". La stigmatisation dont souffrent les Algériens en France ne les incite certainement pas à s'identifier à une nation dont ils se sentent parfois rejetés.

\section{La nouvelle visibilité de l’immigré algérien en France}

Pour appréhender le plus concrètement possible l'ampleur de la stigmatisation, il faut avoir conscience d'une indéniable rupture. Au cours des années soixante-dix, deux phénomènes connexes vont modifier dans l'opinion la perception que la présence sur le sol hexagonal de travailleurs immigrés n'est pas un problème fondamental : la syndicalisation d'une partie des immigrés, la venue en France des familles des immigrés et la volonté de ces derniers d'y demeurer deviennent manifestes. Selon Nicolas Bancel et Pascal Blanchard, "le long processus intégrateur n'opère pourtant plus que partiellement chez des populations fragilisées par la crise économique et par le regard spécifique qu'une société en quête de sens porte sur elle ${ }^{(10)}$.' À cette nouvelle visibilité liée également à l'accroissement de la population algérienne en France (884 320 membres en 1975 et 400000 Français d'origine algérienne - dont les deux tiers sont nés en France - en 1981), à l'émergence et au renforcement d'une véritable élite sociale et culturelle, s'ajoutent en 1973 et 1974 de vives polémiques entre la France et l'Algérie suite à une série d'attentats racistes qui frappent des immigrés algériens et l'arrêt de toute immigration, y compris familiale. De nombreux faits de violence prennent aussi des formes symboliques et cachées (discrimination à l'embauche, au logement, aux loisirs) à l'encontre des immigrés : une véritable stigmatisation s'implante de nouveau en France ${ }^{(11)}$. Tout comme Thomas Deltombe et Laure Pitti, Mathieu Rigouste ${ }^{(12)}$ a bien montré qu'il existe avant et après les indépendances une véritable violence coloniale en métropole sur les populations immigrées issues de l'(ex-)Empire, caractérisée notamment par des conditions d'hébergement difficiles, un chantage des employeurs et une incessante pression policière. 
En France, 1'“identité algérienne" de ces footballeurs ne se construit donc pas simplement à la suite d'un façonnement familial et socioculturel. Dans cette édification du sentiment d'“algérianité", le fait de ne pas se sentir reconnu comme Français durant l'enfance par la société d'accueil ou la société dans laquelle certains sont nés est déterminant. Et que dire des "migrants familiaux", comme l'attaquant Mustapha Dahleb, professionnel de 1969 à 1985, premier d'entre eux à jouer en équipe nationale militaire et civile algérienne, né à Bejaïa en 1952 et qui grandit à Flohimont dans la rigueur des Ardennes où son père est employé dans une tréfilerie, qui doivent signer une "licence d'assimilé" pour jouer et travailler ? Dans un contexte de résurgence de discours xénophobes, beaucoup disent leur souffrance durant l'enfance, l'adolescence : la France ne les perçoit pas entièrement comme "ses enfants". Même Chérif Oudjani, fils d'un célèbre et populaire footballeur professionnel (Ahmed Oudjani) - alors que la majorité des footballeurs "algériens de France", comme le milieu de terrain tlemcénien Abdelghani Djaadaoui (qui arrive en région parisienne à 9 ans en 1956), sont fils d'ouvriers et d'employés dans les principaux bassins industriels -, souligne qu' "ici, en France, on te perçoit comme un Maghrébin".

\section{L'école française, un vecteur puissant de la stigmatisation}

C'est surtout à l'école française que les futurs joueurs ressentent les effets de l'exclusion. Bien qu'Émile Temime et Benjamin Stora rappellent que par "les études secondaires ou universitaires, l'ascension sociale a été plus rapide chez les Algériens que pour toute autre communauté 'étrangère' en France ${ }^{(13)}$,' il n'en demeure pas moins qu'ils subissent presque tous une certaine violence scolaire. Elle émane aussi des insultes de certains camarades. Le milieu de terrain Liazid Sandjak, né en 1966 à Montreuil-sousBois, professionnel au Paris Saint-Germain en 1986 et international algérien l'année suivante, se souvient : “À Noisy-le-Sec où jai grandi, même si au début je n'avais pas d'animosité, je me battais tout le temps à l'école. Parce que souvent, on me traitait de 'sale Arabe."' La suite de ses propos éclaire parfaitement ce repli vers sa culture algérienne : " 'A un moment donné, à force de nous faire sentir qu'on n'est pas des Français, on s'est pris au jeu. Tu te diriges vers ta communauté." Cette violence cachée, plus sournoise, laisse entendre que les immigrés issus de l'ancien empire colonial sont loin d'avoir leur place en France et ne peuvent se fondre dans le "creuset français ${ }^{(14)}$ ". Chagrin et amertume sont exprimés dans le témoignage de Zaïr Kédadouche qui passe son enfance à Aubervilliers : "Cétait la banlieue, la rue, l'affrontement, la magouille, la came, 
le trafic. Quand on chantait des chansons à l'école, dans les poèmes aussi, les héros, les héroïnes étaient toujours blonds aux yeux bleus. Ce n'était jamais moi." Face au sentiment de rejet et d'échec, il est l'un des rares footballeurs professionnels algériens à s'identifier et à participer au "mouvement beur" ("arabe" en verlan) qui naît en France au début des années quatre-vingt ${ }^{(15)}$.

En acceptant de s'engager en équipe nationale algérienne, les footballeurs français d'origine algérienne qui subissent les effets de l'exclusion sociale en France où certains sont nés cherchent à concrétiser symboliquement les fantasmes de leurs parents de réussite et de retour en Algérie. Mais, outre les stratégies sportives de la jeune armée algérienne (qui incorpore de force par exemple Mustapha Dahleb en 1971) face à des joueurs qui cherchent à l'éviter ${ }^{(16)}$, la responsabilité de l'Amicale des Algériens en Europe (AAE), institution tombée dans l'oubli, dans la convocation de certains avec les Fennecs, mérite d'être soulignée.

\section{Le rôle de la Sélection de l'Amicale des Algériens en Europe (AAE) en Algérie}

"Fruit de la dissolution de la fédération de France du Front de libération nationale (FLN), l'Amicale des Algériens de France $(A D A F)$ prend l'appellation d'Amicale des Algériens en Europe' ( $A A E)$ le 20juin 1963. Elle a pour objectif essentiel d'empêcher les Algériens d'opter pour la nationalité française, de fournir des rapports périodiques de renseignements et d'activités et de recenser et surveiller les ex-harkis et les opposants de toute tendance ${ }^{(17) . " ~ V o i l a ̀ ~}$ comment Youssef Fatès présente l'AAE. Après l'utilisation du sport pour l'inculcation d'attitudes nationalistes et l'embrigadement des jeunes émigrés (lutte contre leur "déculturation" en France), elle cherche en France des jeunes sportifs prometteurs parmi la jeunesse émigrée. Par l'organisation de stages et de tournois, par le recensement et la sélection des sportifs binationaux, l'AAE constitue un fichier de joueurs susceptibles d'être utilisés dans le futur pour les équipes nationales algériennes. Avec elle, presque tous les footballeurs professionnels "algériens de France" ont joué en Algérie avant d'être convoqués officiellement par la Fédération algérienne de football. Et ce, d'après Mohamed Harbi, dans un contexte où, en Algérie, "les couches bureaucratiques, économiques, militaires et policières proliferent ${ }^{18}$ ".

Au-delà de la prospection et de l'encadrement de jeunes sportifs algériens en France, le recensement s'apparente à une véritable traque aux espoirs. La "Sélection" réunissant les meilleurs footballeurs amateurs et professionnels se rend en Algérie de 1973 (date de la création de la direction des sports de l'AAE)à 1985. En 1975, Nacer Boughalem, responsable de la direction des sports de l'AAE, déclare que "le regroupement des 
footballeurs algériens opérant en France à l'occasion des journées de l'émigration, lors de la commémoration du 19 juin, a battu l'équipe nationale Espoir par 1 à O". Certes, une équipe de footballeurs professionnels a l'opportunité de jouer contre l'une des équipes nationales. Mais nous constatons de manière plus frappante que la tournée en Algérie de la Sélection en décembre 1976 (pendant la trêve hivernale des clubs français et algériens) fait l'objet d'une importante couverture médiatique. Grâce à une mise en scène savamment orchestrée, "la sélection de l'Amicale des Algériens en Europe en Algérie" est, par exemple, annoncée à plusieurs reprises ; dans la région d'Annaba serait scellé "un acte politico-social par le sport". À travers un long article, le journaliste algérien Hédi Hamel, qui vit en France, relate de manière idyllique la rencontre des deux jeunesses algériennes (locale et "émigrée") et magnifie les objectifs du voyage. Comme les déplacements qui lui succèdent jusqu'au milieu des années quatre-vingt, ses visées sont "culturelles (enrichir les connaissances nationales par des visites d'unités économiques et industrielles) et sportives (prise de contact direct avec le sport national, par des compétitions)".

De plus, avec les nouvelles convocations de footballeurs professionnels en équipe nationale algérienne dès le 28 décembre 1980, cette équipe doit "symboliser l'idée d'une

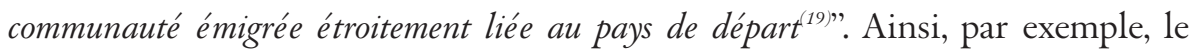
$1^{\mathrm{er}}$ octobre 1980, à Alger, " au cours d'un match de football au profit des sinistrés d'El-Asnam, la sélection des joueurs émigrés concède 4 buts à l'équipe nationale". Notons que "le résultat importait peu. Pour les deux responsables, MM. Chibane et Ben Drama [qui créera en 1984 l'éphémère Union des footballeurs professionnels algériens], 'il s'agit de montrer la ferme volonté des jeunes émigrés de partager la douleur du peuple algérien en des moments pénibles". Pour certains footballeurs "algériens de France", la découverte et le "retour au pays" sont des moments forts. Grâce à cette Sélection, ils vont être amenés à rejoindre les Verts ou à désirer le retour au pays.

\section{Le football, un moyen de satisfaire son désir d'Algérie}

Si la convocation en équipe nationale algérienne se fait, dès décembre 1980, pour des raisons sportives (performances) et ne concerne pas systématiquement tous les footballeurs passés par la Sélection, il est cependant intéressant de considérer qu'elle constitue une véritable passerelle vers les Fennecs. Les sélectionneurs algériens viennent parfois - et très tôt - en France détecter au sein de cette Sélection de jeunes prodiges. Liazid Sandjak présente le système de détection et d'orientation des footballeurs vers l'équipe nationale algérienne : "Concernant cette équipe de 'beurs', on 
reste une semaine à Constantine et à Tizi-Ouzou, et on joue notamment contre l'équipe nationale junior. On la bat 1 à O et je marque le but. Elle était entraînée par l'ancien international, le célèbre Ahcène Lalmas. À la fin du match, il vient me voir et me dit : 'Viens en équipe d'Algérie, ce sera toi notre avant-centre.'Je suis donc revenu dans la vraie équipe nationale junior." Mais cette Sélection ne peut être vue uniquement comme une filière sportive. Elle renforce également le sentiment des joueurs d'être des Algériens et devient un temps de "socialisation secondaire" à l'Algérie. En effet, à travers ces voyages sportifs, ils découvrent leurs racines et le lien avec l'Algérie se renforce.

Le défenseur monégasque Abdallah Liegeon Medjadi, né en 1957 à Oran, qui a quitté Tiaret en feu et la guerre d'Algérie encore enfant entre sa mère et un militaire français pour s'installer dans un quartier H.L.M. de Lons-le-Saunier, décrit son émotion : "Le but, c'était de pouvoir remettre les pieds dans mon pays. C'était extraordinaire, un cadeau. Dès qu'on a ouvert la porte de l'avion, je me suis dit : 'je suis dans mon pays'. C'est peut-être l'un des moments les plus importants de ma vie." La même émotion traverse Nordine Kourichi : "Les rencontres assidues organisées par l'Amicale nous ont permis de cultiver ce lien avec l'Algérie."

Cette Sélection sportive de l'Amicale constitue un temps de "socialisation secondaire" et une filière sportive pour l'équipe nationale algérienne. Mais, contrairement à ce que prétendent les publications officielles de l'Amicale qui louent leur "ambition du retour", il n'a jamais été question pour ces champions qui ont grandi en France de venir s'installer - même provisoirement - en Algérie.

\section{Conclusion}

Ce désir de représenter l'Algérie chez ces footballeurs algériens "migrants familiaux" et "nés en France" se construit donc sur un façonnement socioculturel et sur des stratégies personnelles indéniables, sportives et extra-sportives. En France, le façonnement socioculturel de l'identité algérienne passe systématiquement par la famille et très souvent par l'évolution au sein de la Sélection de l'AAE. Ce désir d'aller jouer provisoirement de l'autre côté de la Méditerranée peut s'expliquer en partie par les discriminations ressenties et réelles dans le pays où ils grandissent, la France. Ce "désir d'Algérie" ne repose pas sur un espoir d'ascension socio-économique - leurs salaires en France sont plus importants que leurs gratifications non systématiques avec les Verts. D’une part, il répond à un souhait des footballeurs algériens de participer à des compétitions internationales prestigieuses (ce qui leur ouvre les portes de clubs renommés) alors qu'ils savent qu'ils ne peuvent pas prétendre à une place de titulaire en équipe de France. D'autre part, ce désir est aussi lié à l'exemption (ou non) 
des joueurs de leurs obligations militaires ; les footballeurs cherchent à incarner en Algérie et en France la réussite de leurs parents et grands-parents dont le départ vers la métropole pèse sur leur histoire familiale.

Connaître les conditions de possibilité du "désir d'Algérie" des footballeurs "algériens de France" ainsi que leur condition d" immigré sportif" sous le maillot des Fennecs s'avère indispensable après la Coupe du Monde 2010 en Afrique du Sud, où 17 des 23 joueurs (soit 73,9\%) convoqués par le sélectionneur algérien Rabah Saadane sont nés en France. Plus que jamais, des rapports de domination post-coloniale entre ces deux pays semblent persister ; l'Algérie indépendante apparait sous la dépendance sportive de la France et de ses centres de formation.

\section{Bibliographie}

- Bancel Nicolas et Blanchard Pascal, De l'indigène à l'immigré, Paris, Gallimard, 1998.

- Boli Claude, Gastaut Yvan et Grognet Fabrice, Allez la France, Football et immigration, Paris,

Gallimard-CNHI, 2010.

- Chebel Malek, Les Symboles de l'islam, Paris, Assouline, 1997.

- Deltombe Thomas, L'Islam imaginaire : la construction médiatique de lislamophobie en France 1975-2005, Paris, La Découverte, 2005.

- Dubet François et Lapeyronnie Didier, Les Quartiers d'exil, Paris, Seuil, 1992.

- Ernault Gérard, Dahleb : le défi de Paris, Paris, Calmann-Lévy, 1977.

- Falcoz Marc et Koebel Michel, Intégration par le sport : représentations et réalités, Paris, L'Harmattan, 2005.

- Fatès Youssef, "La politique centrifuge d'intégration des jeunes par le sport de l'Amicale des Algériens en France", in Falcoz Marc et Koebel Michel, Intégration par le sport : représentations et réalités, L'Harmattan, Paris, 2005.

- Frenkiel Stanislas, "Les footballeurs du FLN : des patriotes entre deux rives", in Migrations et Société, $\mathrm{n}^{\circ} 110,2007$.

- Frenkiel Stanislas, "Lélaboration du projet migratoire des footballeurs algériens (1944-1962)", in Migrance, $\mathrm{n}^{\circ} 29,2008$.

- Frenkiel Stanislas, "Des footballeurs professionnels algériens entre deux rives - Travailler en France, jouer pour l'Algérie (1954-2002)", thèse STAPS, Université Paris-Sud-11, 2009.

- Guenif Souilamas Nacira, Des "Beurettes” aux descendantes d'immigrants nord-africains, Paris, Grasset, 2000.

- Grandguillaume Gilbert, "Langue, identité et culture nationale au Maghreb", in Référence : peuples méditerranéens, $\mathrm{n}^{\circ} 9,1979$.

- Hamel Hédi, La Grande Aventure du football algérien, Alger-Bruxelles, Enal-Gam, 1984.

- Harbi Mohamed, Le FLN, mirage et réalité, Paris, Éditions J.A., 1980.

- Lanfranchi Pierre et Taylor Matthew, Moving with the Ball, Oxford, Berg, 2001.

- Noiriel Gérard, Le Creuset français - Histoire de limmigration, XIXe-XXe siècles, Paris, Seuil, 1988. 
- Pitti Laure, "La main-d'ceuvre algérienne dans l'industrie automobile ou les oubliés de l'histoire", in Kadri Aïssa et Prévost Gérard, Mémoires algériennes, Paris, Syllepse, 2004.

- Poli Raffaele, Le Marché des footballeurs, Berne, Peter Lang, 2010.

- Rigouste Mathieu, L'Ennemi intérieur, des guerres coloniales au nouvel ordre sécuritaire, Paris, La Découverte, 2008.

- Sayad Abdelmalek, "Les trois 'âges' de l'émigration algérienne en France", in Actes de la recherche en sciences sociales, $\mathrm{n}^{\circ} 15,1977$.

- Scagnetti Jean-Charles, "Coupe du monde 1982 : les internationaux algériens et leur équipe nationale", in Migrance, $n^{\circ} 29,2008$.

- Schor Ralph, Histoire de l'immigration de la fin du XIX siècle à nos jours, Paris, Armand Colin, 1996.

- Stora Benjamin et Temime Émile, "L'immigration algérienne", in Gervereau Laurent, Milza Pierre et Temime Émile, Toute la France - Histoire de l'immigration en France au XXe siècle, Paris, Somogy, 1998.

\section{Notes}

1. Lanfranchi Pierre et Taylor Matthew, Moving with the Ball, Oxford, Berg, 2001.

2. Boli Claude, Gastaut Yvan et Grognet Fabrice, Allez la France, Football et immigration, Paris, Gallimard-CNHI, 2010 ; Frenkiel Stanislas, "Les footballeurs du FLN : des patriotes entre deux rives", in Migrations et Société, n 110, 2007 et "L'élaboration du projet migratoire des footballeurs algériens (1944-1962)", in Migrance, n 29, 2008 ; Poli Raffaele, Le marché des footballeurs, Berne, Peter Lang, 2010.

3. Falcoz Marc et Koebel Michel, Intégration par le sport : représentations et réalités, Paris, L'Harmattan, 2005.

4. Scagnetti Jean-Charles, "Coupe du monde 1982 : les internationaux algériens et leur équipe nationale", in Migrance, $n^{\circ} 29,2008$.

5. Guenif Souilamas Nacira, Des "beurettes" aux descendantes d'immigrants nord-africains, Paris, Grasset, 2000.

6. Grandguillaume Gilbert, "Langue, identité et culture nationale au Maghreb", in Référence :peuples méditerranéens, $\mathrm{n}^{\circ}$ 9, 1979 .

7. Chebel Malek, Les Symboles de l'islam, Paris, Assouline, 1997.

8. Stora Benjamin et Temime Émile, "L'immigration algérienne", in Gervereau Laurent, Milza Pierre et Temime Émile, Toute la France - Histoire de l'immigration en France au XX' siècle, Paris, Somogy, 1998.

9. Sayad Abdelmalek, "Les trois 'âges' de l'émigration algérienne en France", in Actes de la recherche en sciences sociales, $\mathrm{n}^{\circ} 15,1977$.

10. Bancel Nicolas et Blanchard Pascal, De l'indigène à l'immigré, Paris, Gallimard, 1998.

11. Schor Ralph, Histoire de l'immigration de la fin du XIX'e siècle à nos jours, Paris, Armand Colin, 1996.

12. Voir Deltombe Thomas, L'Islam imaginaire : la construction médiatique de l'islamophobie en France 1975-2005, Paris, La Découverte, 2005 ; Rigouste Mathieu, L'Ennemi intérieur, des guerres coloniales au nouvel ordre sécuritaire, Paris, La Découverte, 2008 ; Pitti Laure, "La main-d'ceuvre algérienne dans l'industrie automobile ou les oubliés de l'histoire", in Kadri Aïssa et Prévost Gérard, Mémoires algériennes, Paris, Syllepse, 2004.

13. Stora Benjamin et Temime Émile, "L'immigration algérienne", op. cit.

14. Noiriel Gérard, Le Creuset français - Histoire de l'immigration, $X I X^{e}$-XXe siècles, Paris, Seuil, 1988.

15. Dubet François et Lapeyronnie Didier, Les Quartiers d'exil, Paris, Seuil, 1992.

16. Frenkiel Stanislas, "Des footballeurs professionnels algériens entre deux rives - Travailler en France, jouer pour l'Algérie (1954-2002)", thèse STAPS, Université Paris-Sud-11, 2009.

17. Fatès Youssef, "La politique centrifuge d'intégration des jeunes par le sport de l'Amicale des Algériens en France", in Falcoz Marc et Koebel Michel, Intégration par le sport : représentations et réalités, Paris, L'Harmattan, 2005.

18. Harbi Mohamed, Le FLN, mirage et réalité, Paris, Éditions J.A., 1980.

19. Scagnetti Jean-Charles, "Coupe du monde 1982 : les internationaux algériens et leur équipe nationale", in Migrance, $\mathrm{n}^{\circ} 29,2008$. 